\title{
Intra-Arterial Embolization as a Treatment Option in a Case of GAVE Syndrome
}

\author{
Soumil Singhal ${ }^{1, \oplus}$ Mangerira C. Uthappa ${ }^{1, \oplus}$ \\ ${ }^{1}$ Department of Intervention Radiology and Intervention \\ Oncology, BGS Gleneagles Global Hospital Bangalore, Bangalore, \\ Karnataka, India
}

\begin{abstract}
Address for correspondence Soumil Singhal, MD, Department of Intervention Radiology and Intervention Oncology, BGS Gleneagles Global Hospital, 67, Uttarahalli Main Road, Sunkalpalya, Kengeri, Bangalore 560060, India (e-mail: drsoumilsinghal75@gmail.com).
\end{abstract}
Abstract
Keywords
- stomach
- hemorrhage
- embolization
- intra-arterial therapy

Gastric antral vascular ectasia (GAVE) syndrome is a rare condition affecting the stomach. It is associated with upper gastrointestinal bleeding. The exact pathogenesis of the condition is poorly understood. Watermelon stomach is synonymous with GAVE syndrome due to its endoscopic appearance. Here we report a unique experience of performing an intra-arterial embolization in a case of GAVE presenting with uncontrolled bleeding.

\section{Introduction}

Gastric antral vascular ectasia syndrome or GAVE syndrome is a rare condition of the stomach, which is associated with severe acute or chronic upper gastrointestinal bleed. The exact pathogenesis of the condition is poorly understood; however, the condition has been linked to various medical conditions.

This condition was first described by Rider et al in $1953 .{ }^{1}$ Wheeler et $\mathrm{al}^{2}$ reported its characteristic endoscopic features. Based on the distinctive endoscopic features, the term watermelon stomach was coined by Jabbari et al. ${ }^{3}$ Watermelon stomach endoscopically resembles a pattern similar to the watermelon's exterior, the prominent erythematous stripe-like pattern radiates from the pylorus to the antrum resembling the similar design. GAVE syndrome is often misdiagnosed as either antral gastritis or portal hypertensive gastropathy. ${ }^{4}$ Treatment of the condition can be challenging and mainly includes three options including: (1) medical management, (2) endoscopic management, and (3) surgical management. Scanty case reports of the use of various pharmacologic therapies in the management of the condition have been reported. Surgical resection is the definitive option available, but it comes at the cost of significant morbidity and mortality rates. ${ }^{4}$ Endoscopic management is the main conservative treatment option available. ${ }^{5}$ Complete eradication of the condition requires two to four sessions based on the site, extent, and number of lesions. Up to $40 \%$ of postprocedure recurrence has been reported in some studies. ${ }^{6}$ We report a unique experience of performing an intra-arterial embolization in a case of GAVE presenting with uncontrolled bleeding even after multiple rounds of endoscopic management.

\section{Case Report}

A 63-year-old male patient, who is a known case of chronic liver disease (etiology: cryptogenic) since 2011, presented to the emergency department with complaints of two episodes of hematemesis and melena. The patient had several such incidents in the past for which esophageal variceal ligation and argon plasma coagulation (APC) was performed on several occasions.

On examination, patient's vitals were stable with evidence of pallor, pedal edema, and ascites. The patient had a Child-Pugh score B with a MELD (Model for End-Stage Liver Disease) score of 15 . Supportive measures were taken, and an emergency upper gastrointestinal endoscopy was performed considering bleeding varices as the cause for the patient's symptoms. Endoscopy showed eradicated esophageal varices, mild portal gastropathy in fundus and body, nodular erythematous streaks in the antrum extending to the proximal body, and lesion in the prepyloric region and the antrum with active ooze. Duodenum showed features of erythematous duodenopathy with no active ooze. A diagnosis of GAVE syndrome was made. Forced argon plasma coagulation (APC) was performed ( $40 \mathrm{~W}, 2 \mathrm{~L} / \mathrm{min}$ ) to settle down the active ooze. The patient was symptomatically normal for 24 hours following which he redeveloped melena with a drop
License terms

(요 (1) $\Theta \circledast$ 
in hemoglobin concentration (drop from 9 to $8.3 \mathrm{~g} \%$ ). Due to the sudden drop, again an emergency repeat endoscopy was performed, which showed blood at the former site of APC. A definite bleeding point could not be found due to blood in the field of view. APC was abandoned, and computed tomography (CT) was planned. A CT was performed after a multidisciplinary meeting between the hepatologist, hepatic surgeon, and the interventional radiology team.

Computed tomography abdominal angiography was performed (16 slice, GE Healthcare). The scan was performed in the supine position in suspended respiration. The contrast was injected using a pressure injector through an 18 gauge cannula sited in the left upper limb vein. Omnipaque (Iohexol 350, General Electric Healthcare) was used as intravenous contrast media. Patient underwent two angiographic phases with the first phase acquisition taken at 6 seconds and the second phase at 45 seconds following a bolus trigger. Technical parameters were set as pitch (1.2), collimation (arterial: $16 \times 0.6 \mathrm{~mm}$ and enous: $24 \times 1.2 \mathrm{~mm}$ ), rotational time (0.6 s), kV (120), and mA (120). CT angiography showed the patient to have features of chronic liver disease with features of portal hypertension and ascites. There was a focal mucosal thickening seen at the antral region of the stomach with multiple prominent vessels supplying this area with mildly increased vascularity best appreciated on the arterial phase (-Figs. 1, 2). No active contrast extravasation was
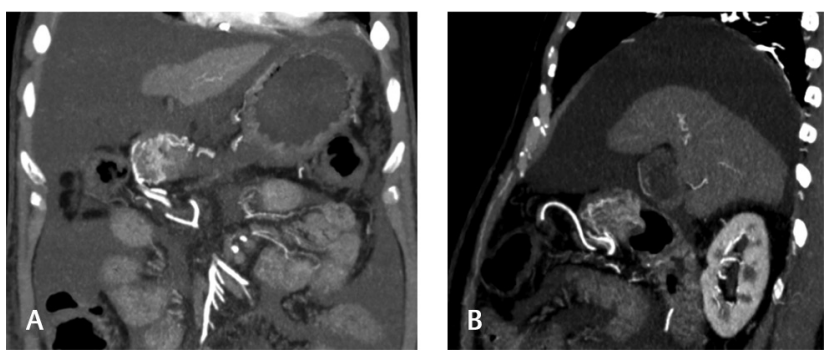

Fig. 2 Contrast-enhanced CT abdomen, coronal, and sagittal reformat images ( $\mathbf{A}$ and $\mathbf{B}$ ) showing mild focal mucosal thickening at the antral-pyloric region of the stomach with multiple prominent vessels supplying this area with mildly increased vascularity.
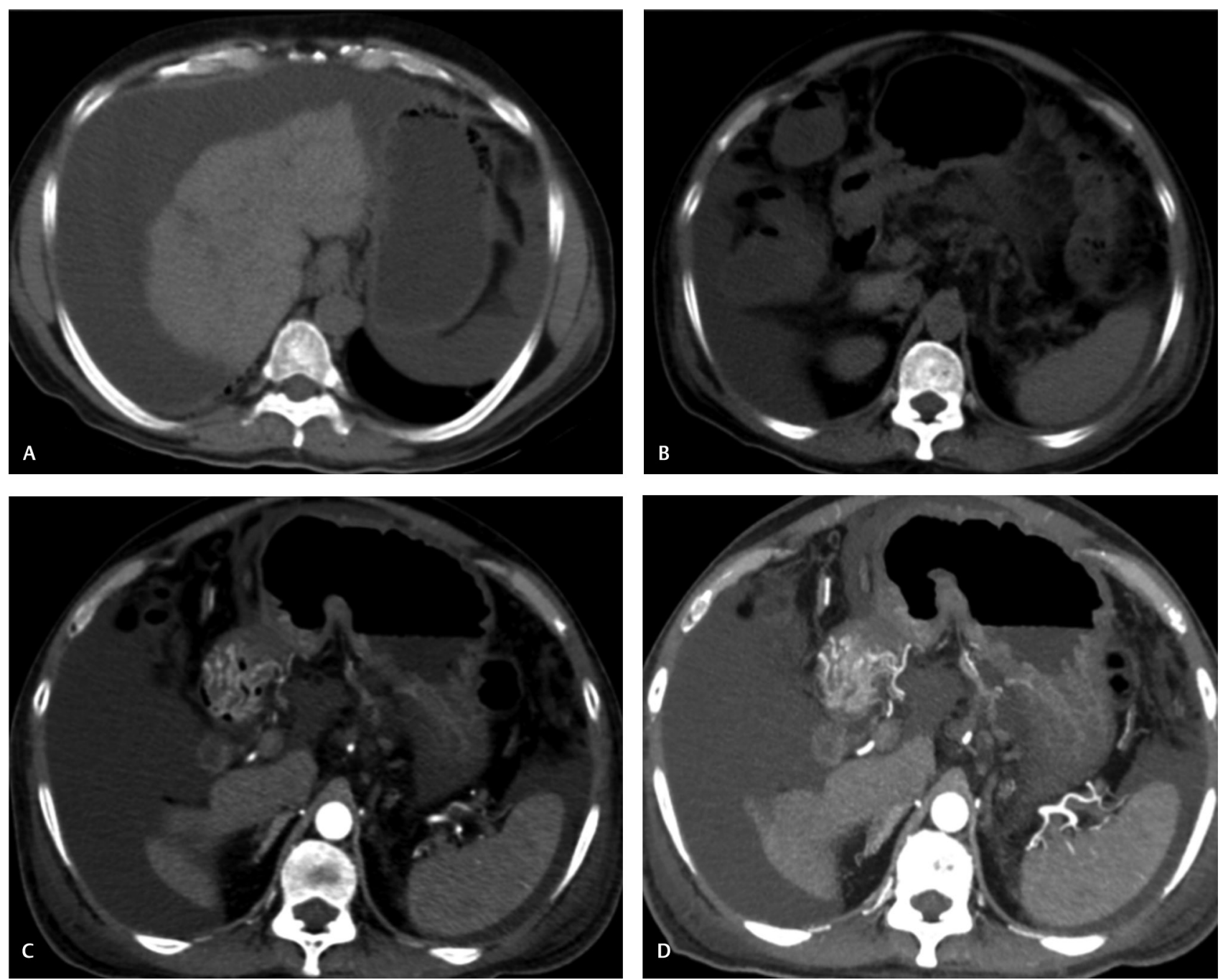

Fig. 1 Contrast-enhanced CT abdomen, axial images. (A) Noncontrast scan showing features of chronic liver disease with ascites, (B) section across the pyloric region of stomach, $(\mathbf{C})$ mild focal mucosal thickening at the antral-pyloric region of the stomach with multiple prominent vessels supplying this area with mildly increased vascularity, and (D) MIP image of the corresponding region showing multiple prominent vessels supplying the antrum-pyloric region. MIP, maximum intensity projection. 
demonstrated on any phase of the CT angiography. Due to recurrent fall in patients hemoglobin concentration, established the diagnosis of GAVE syndrome and failed APC, a unique and alternative approach was planned for the management of the condition. An empirical intra-arterial embolization was proposed based on the $\mathrm{CT}$ angiographic finding of multiple arterial feeders to the thickened antral region.

A super selective empirical intra-arterial embolization was planned and performed under mild sedation. Right femoral arterial access was achieved under ultrasound guidance, and a $5 \mathrm{~F}$ sheath was inserted. Celiac artery and common hepatic artery were cannulated using a $5 \mathrm{~F}$ cobra catheter, and angiographic run was acquired. A normal celiac artery anatomy was noted with the common hepatic artery diving into hepatic artery proper and gastroduodenal artery. Super selectively catheterization of the gastroduodenal artery was then performed using a 2.7F microcatheter (Progreat, Terumo). An angiographic phase was acquired, which demonstrated multiple small vessels supplying the area of focus in the antral region with these small gastric feeding vessels arising mainly from the right gastroepiploic artery with early contrast runoff ( - Fig. 3). Empirical embolization was performed using gel foam slurry, which was made by mixing small gelatin sponge pledgets with contrast using the Tessari technique. Gel foam slurry was first injected super-selectively into these multiple small feeding vessels. Check angiography showed stasis of flow across these small feeding vessels. Following this, coil embolization was performed using three Nester micro-coils $(2-70 \times 4 \mathrm{~mm}$ and $1-140 \times 4 \mathrm{~mm}$ ), which was placed in the right gastroepiploic artery and the superior pancreaticoduodenal artery, respectively ( - Fig. 4). Cross circulation from the superior mesenteric artery was blocked using gel foam slurry injection into the inferior pancreaticoduodenal artery, and this was confirmed on the successive angiographic run. Other sources of cross circulation were checked by confirming angiographic series from the proper hepatic artery, gastric artery, splenic artery, and colic arteries. The patient was hemodynamically stable postprocedure with no further drop in hemoglobin concentration or any complaints of melena. The patient was shifted to ICU on day 5 due to development of oliguria, increase lactate, and breathlessness. The patient developed multi-organ failure and bacterial peritonitis by
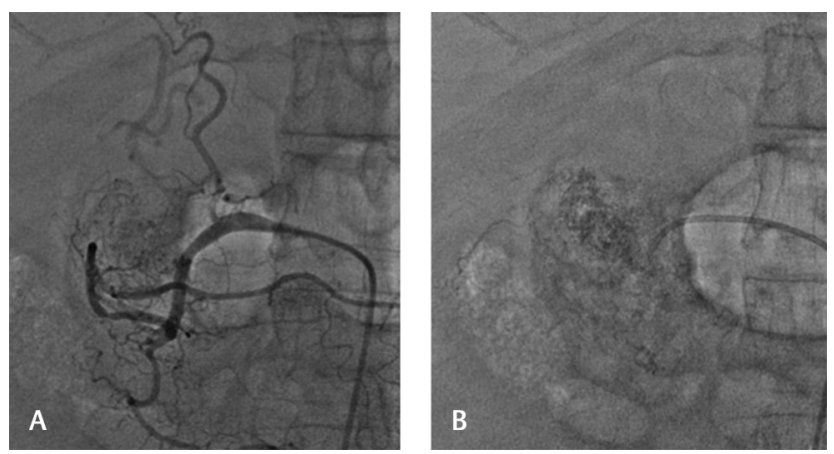

Fig. 3 Conventional angiography images (A and $\mathbf{B}$ ), contrast blush with the early draining vein in the antral-pyloric region of the stomach with feeder vessels arising from the right gastroepiploic artery.
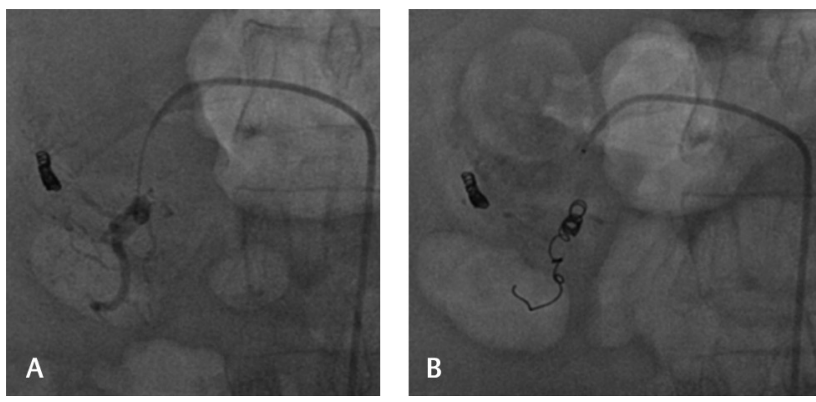

Fig. 4 Postembolization images (A and $\mathbf{B})$.

day 7 and eventually succumbed due to cardiopulmonary arrest. No follow-up imaging or endoscopy was performed due to deterioration in the patients' condition.

\section{Discussion}

GAVE syndrome or watermelon stomach is a rare condition of the stomach, which can be misinterpreted as either antral gastritis or portal hypertensive gastropathy. ${ }^{4}$ The condition is common in elderly patients with a female preponderance. ${ }^{7}$ The mean age at presentation is about 73 years in women and 68 in men, with up to $71 \%$ of patients being women. ${ }^{8}$ Ninety percent of patients present with nonresponsive iron deficiency anemia and occult bleed. ${ }^{9}$ The exact pathogenesis is not well understood; however, the condition has shown a link to various conditions like liver cirrhosis, diabetes, end-stage renal disease, hypothyroidism, connective tissue disorder, and cardiac disease. ${ }^{1,710}$ Cirrhosis is one of the most common causes of the condition, with up to $30 \%$ of patients having preexisting cirrhosis. ${ }^{1,10}$ Patients with GAVE present with chronic iron-deficiency anemia, acute GI bleed, unexplainable abdominal pain, and rarely as gastric outlet obstruction. ${ }^{11}$ Up to $60 \%$ of patients with connective tissue disorder have skin telangiectasias. ${ }^{12}$ Diagnosis of the condition can be using endoscopy, enteroscopy, and sometimes using red blood cell scans or capsule endoscopy. GAVE syndrome, classically present as a red lesion which is often hemorrhagic, which can be either in a linear fashion or diffusely spread out. These are predominantly located within the gastric antrum. ${ }^{13}$ Histologically, the underlying region shows hyperplastic antral mucosa, thrombosed dilated capillary channels, and hypertrophy of the fibromuscular lamina propria without inflammatory signs. ${ }^{14}$ The histological findings are also appreciated on endoscopic ultrasound. Some studies have found a 5-year latency period for the diagnosis of the condition. ${ }^{15}$ Due to its features, GAVE can be misinterpreted as portal hypertensive gastropathy; however, there are specific features which help in differentiating the conditions. Portal hypertensive gastropathy involves the corpus and the fundal region of the stomach, whereas, GAVE is most limited mainly to the antral region. GAVE has a classical endoscopic finding. Procedures such as $\beta$-blocker therapy or transjugular intrahepatic shunt are not helpful in case of GAVE syndrome. ${ }^{1,10,16,17}$

Treatment of the condition can be challenging and mainly includes three options including, (1) medical management, 
(2) endoscopic management, and (3) surgical management. Medical management consists of the use of various pharmacological agents, such as steroids, octreotide, estrogen-progesterone hormonal combination therapy, tranexamic acid, thalidomide, cyproheptadine, $\alpha$-interferon, and calcitonin. ${ }^{1,10}$ The effectiveness of medical management has only been reported in case reports/case series and need randomized control trials to find efficacy and drug safety. ${ }^{18}$ Endoscopic treatment is a preferred treatment option and includes band ligation, cryotherapy, argon plasma coagulation, neodymium-yttrium-aluminum-garnet laser coagulation, radiofrequency ablation, and sclerotherapy. ${ }^{11,18}$ APC is the treatment of choice in patients with GAVE and has shown effective results in both bleeding and rebleeding. ${ }^{18}$ Surgical treatment is reserved as a last resort in these cases; however, it comes at the cost of significant morbidity and mortality rates. ${ }^{4}$ No controlled studies have been performed to suggest if medical-surgical treatment options are superior. Studies have suggested the use of surgical interventions in medically refractory cases. ${ }^{16}$

Transarterial embolization has not been well established as a treatment option. Jabbari et $\mathrm{al}^{3}$ who first coined the term watermelon stomach failed to demonstrate the lesion on angiography in three patients. It was suggested that the failure to detect GAVE on angiography could be subtle and hence missed due to early arterial filling and emptying which would appear normal in the late venous phase. Second, the surrounding hypervascularity can also obscure the lesion. ${ }^{19}$ Digital angiography allows better visualization of the otherwise subtle lesion. Terawaki et al reported a productive and successful embolization in the case of GAVE syndrome. ${ }^{20}$ Another case report of successful embolization was published in Japan for refractory GAVE. ${ }^{21}$ Complications of embolization include necrosis and gastric wall ulcerations. ${ }^{22}$

\section{Conclusion}

We report a unique medical experience of performing an endovascular embolization in a case of GAVE which has rarely been published in the past as per our literature review. Embolization provides an additional treatment option in patients who are not manageable with endoscopy or have several rebleed episodes or who do not want surgery as a treatment option.

\section{Conflicts of Interest}

None declared.

\section{Reference}

1 Rider JA, Klotz AP, Kirsner JB. Gastritis with veno-capillary ectasia as a source of massive gastric hemorrhage. Gastroenterology 1953;24(1):118-123

2 Wheeler MH, Smith PM, Cotton PB, Evans DMD, Lawrie BW. Abnormal blood vessels in the gastric antrum: a cause of upper-gastrointestinal bleeding. Dig Dis Sci 1979;24(2):155-158
3 Jabbari M, Cherry R, Lough JO, Daly DS, Kinnear DG, Goresky CA. Gastric antral vascular ectasia: the watermelon stomach. Gastroenterology 1984;87(5):1165-1170

4 Sebastian S, O'Morain CA, Buckley MJM. Review article: current therapeutic options for gastric antral vascular ectasia. Aliment Pharmacol Ther 2003; 18(2):157-165

5 Jensen DM, Chaves DM, Grund KE. Endoscopic diagnosis and treatment of watermelon stomach. Endoscopy 2004;36(7):640-647

6 Yusoff I, Brennan F, Ormonde D, Laurence B. Argon plasma coagulation for treatment of watermelon stomach. Endoscopy 2002;34(5):407-410

7 Stefanidis I, Liakopoulos V, Kapsoritakis AN, et al. Gastric antral vascular ectasia (watermelon stomach) in patients with ESRD. Am J Kidney Dis 2006; 4 7(6):e77-e82

8 Gostout CJ, Viggiano TR, Ahlquist DA, Wang KK, Larson MV, Balm R. The clinical and endoscopic spectrum of the watermelon stomach. J Clin Gastroenterol 1992;15(3):256-263

9 Appleyard MN, Swain CP. Endoscopic difficulties in the diagnosis of upper gastrointestinal bleeding. World J Gastroenterol 2001;7( 3):308-312

10 Selinger CP, Ang YS. Gastric antral vascular ectasia (GAVE): an update on clinical presentation, pathophysiology and treatment. Digestion 2008; 77(2):131-137

11 Nguyen H, Le C, Nguyen $\mathrm{H}$. Gastric antral vascular ectasia (watermelon stomach)-an enigmatic and often-overlooked cause of gastrointestinal bleeding in the elderly. Perm J 2009;13(4):46-49

12 Johnson J, Derk CT. Gastric antral vascular ectasia in systemic sclerosis. Int J Rheumatol 2011; 2011:305238

13 Burak KW, Lee SS, Beck PL. Portal hypertensive gastropathy and gastric antral vascular ectasia (GAVE) syndrome. Gut 2001;49(6):866-872

14 Suit PF, Petras RE, Bauer TW, Petrini JL Jr. Gastric antral vascular ectasia. a histologic and morphometric study of "the watermelon stomach." Am J Surg Pathol 1987; 11(10):750-757

15 Tsai HH, Smith J, Danesh BJ. Successful control of bleeding from gastric antral vascular ectasia (watermelon stomach) by laser photocoagulation. Gut 1991;32(1):93-94

16 Novitsky YW, Kercher KW, Czerniach DR, Litwin DE. Watermelon stomach: pathophysiology, diagnosis, and management. J Gastrointest Surg 2003;7(5):652-661

17 Dulai GS, Jensen DM, Kovacs TO, Gralnek IM, Jutabha R. Endoscopic treatment outcomes in watermelon stomach patients with and without portal hypertension. Endoscopy 2004;36(1):68-72

18 Hsu WH, Wang YK, Hsieh MS, et al. Insights into the management of gastric antral vascular ectasia (watermelon stomach) Therap Adv Gastroenterol 2018;11:X17747471

19 Robertson IR, Tait NP, Jackson JE. Vascular ectasia of the gastric antrum: angiographic findings. AJR Am J Roentgenol 1996;166(1):87-89

20 Terawaki H, Kobayashi S, Kato J, et al. A case report of hemodialysis patient who presented recurrent bleeding from gastric antral vascular ectasia (GAVE) associated with liver cirrhosis: the effectiveness of trans-arterial embolization to bilateral gastric arteries. J Jpn Soc Dial Ther. 2008; 41:803-808

21 Yoshida H, Takahashi H, Akoshima H, et al. Successful treatment of refractory gastric antral vascular ectasia using transcatheter arterial embolization. Clin J Gastroenterol 2013;6(3):231-236

22 Ashiya E, Yagi N, Shiomi H, et al. A case report of gastric vascular ectasia patient successfully treated by trans-arterial embolization despite the inefficiency of argon plasma coagulation. Jpn J Gastroenterol 2003;100:A304 